\title{
\begin{tabular}{l|l} 
POLITIQUES \& & Politiques et management public
\end{tabular}

\section{La fabrication du « médico-économique » à l'hôpital français : une analyse de la littérature}

The integration of medical and economic dimensions of the activities in french hospitals: analysis of french academics papers

Annick Valette, Corinne Grenier, Olivier Saulpic et Philippe Zarlowski

\section{OpenEdition}

Journals

Édition électronique

URL : http://journals.openedition.org/pmp/9379

ISSN : 2119-4831

Éditeur

Institut de Management Public (IDPM)

\section{Édition imprimée}

Date de publication : 1 juillet 2015

Pagination : 241-264

ISBN : 978-2-7430-2138-2

ISSN : 0758-1726

\section{Référence électronique}

Annick Valette, Corinne Grenier, Olivier Saulpic et Philippe Zarlowski, « La fabrication du « médicoéconomique » à l'hôpital français : une analyse de la littérature », Politiques et management public [En ligne], Vol 32/3 | 2015, mis en ligne le 21 juin 2019, consulté le 24 janvier 2021. URL : http:// journals.openedition.org/pmp/9379 


\section{Annick Valette ${ }^{a *}$, Corinne Grenier ${ }^{b}$, Olivier Saulpic ${ }^{c}$ et Philippe Zarlowskic}

a IAE, Université Grenoble-Alpes, BP 47, 30140 Grenoble Cedex 9

${ }^{b}$ KEDGE Business School, Domaine de Luminy, Rue Antoine Bourdelle, 13009 Marseille 'ESCP Europe, 79 Avenue de la République, 75011 Paris

\section{Résumé}

Dans cet article, nous conduisons une analyse de 67 articles et communications issus de travaux de recherche en gestion qui se sont intéressés à la mise en œuvre des réformes à I'hôpital public depuis 2005. Nous regardons ce qu'ils nous apprennent sur un enjeu clé de ces réformes à savoir l'intégration des dimensions médicales et économiques de l'activité à l'hôpital. Nos résultats montrent que ces travaux de recherche ne traitent pas de la construction du médico-économique lui-même ou des conséquences des réformes sur les pratiques médico-économiques des acteurs, mais plutôt des difficultés de mise en œuvre de ces réformes. Dans les travaux analysés, le médico-économique apparaît donc comme une donnée ou un élément de contexte, plutôt que comme un enjeu ou un objet de recherche en soi. L'étude de notre corpus fait notamment ressortir une place centrale pour l'instrumentation de gestion dans les changements en cours, et une grande variété dans les pratiques des établissements, en dépit du caractère standard des réformes. Au-delà des résultats des travaux de recherche, cette revue de littérature nous conduit à formuler des propositions pour renforcer la structuration de la recherche appliquée sur le management hospitalier, et développer les contributions de la recherche à l'élaboration des politiques.

(c) 2015 IDMP/Lavoisier SAS. Tous droits réservés

Mots clés : hôpital, réformes, tarification à l'activité, gouvernance, design organisationnel, outils de gestion, pôles.

*Auteur correspondant : annick.valette@upmf-grenoble.fr

doi :10.3166/pmp.32.241-264 @ 2015 IDMP/Lavoisier SAS. Tous droits réservés 


\section{Abstract}

The integration of medical and economic dimensions of the activities in french hospitals: analysis of french academics papers. We look at what we learn on one key issue of these reforms i.e. the integration of medical and economic dimensions of the activities in hospitals. Our analysis shows that these academic papers do not focus on the construction of medico-economic logic or the consequences of these reforms on medico-economic practices of actors, but rather on the difficulties of implementing the reforms. The medicoeconomic aspect is seen as a fact or as a contextual element rather than a challenge (or an issue?) or an object of research in itself. The study of our data highlights the central role of managerial instrumentation in the ongoing changes and a large variety of the practices in establishments, in spite of the reforms that promote standardization. Beyond these results, this literature review leads us to formulate proposals to strengthen the structuring of research on hospital management, and to make possible that research contributions which may feed public policy elaboration.

(c) 2015 IDMP/Lavoisier SAS. Tous droits réservés

Keywords: hospital, reforms, activity-based tariff, governance, organizational design, managerial tools, clinical units.

On peut dire qu'il y avait deux mondes inconciliables. Les directeurs d'hôpitaux avec leur culture budgétaire, juridique et de santé publique, les médecins porteurs de leur connaissance médicale, ancrées dans la relation patient-malade. Quand l'hôpital a été mis sous contraintes, tout le monde s'est dit qu'il fallait arrêter la schizophrénie (...) On parie sur la maîtrise médicalisée des dépenses contre la maîtrise comptable. (Permanent F.H.F., avril 2010)

\section{Introduction}

Traditionnellement, l'activité des établissements de soins a été décrite comme le produit de plusieurs logiques institutionnelles (Kitchener, 2002, Arndt et Bigelow, 2006) ou de plusieurs « mondes » (Glouberman et Mintzberg, 2002). « Alors que la logique médicosoignante place le traitement et le soin du patient avant toute considération économique, la logique économique prône la mutualisation des coûts et la définition des activités en fonction des recettes » (Grenier et Bernardini, 2015, p. 126). La logique médicale est traditionnellement portée par les médecins et les autres soignants, la logique économique par les administratifs. En France, plus qu'ailleurs, l'accès réservé aux fonctions de directeurs d'établissement au corps des directeurs d'hôpitaux a pu contribuer à construire une étanchéité entre les deux logiques. Le renforcement au cours des dix dernières années de la contrainte budgétaire sur des dépenses de santé structurellement croissantes a motivé la mise en œuvre de politiques visant à transformer l'environnement institutionnel et l'organisation interne des hôpitaux. Ainsi, depuis le milieu des années 2000, plusieurs réformes importantes ont cherché à intégrer davantage l'économique et le médical, à créer les conditions d'une prise en compte accrue, par les acteurs administratifs comme médicaux, de l'impact économique de leurs décisions comme de leurs pratiques médico- 
soignantes (Domy et al., 2014). Ces réformes portent d'abord sur la généralisation rapide, entre 2004 et 2008, de la tarification à l'activité (T2A) pour déterminer les recettes des hôpitaux pour les activités de médecine, chirurgie et obstétrique (loi n 2003-1199 du 18 décembre 2003 de financement de la sécurité sociale pour 2004). Ensuite, en termes de gouvernance, les réformes visent à une association accrue des médecins à la gestion interne des établissements à travers notamment la création de centres de responsabilités, les pôles, dirigés par un médecin (« Nouvelle gouvernance » hospitalière, correspondant à l'un des volets du Plan hôpital 2007 de l'ordonnance du 2 mai 2005) et à un renforcement en parallèle des pouvoirs et responsabilités des directeurs d'établissements (ordonnance du 2 mai 2005 prolongée par la loi Hôpital Patients Santé Territoires dite « loi Bachelot» du 21 juillet 2009). "Réconcilier» ou mieux associer au sein des établissements les logiques administratives et médicales et les acteurs qui les portent devait permettre de créer les conditions d'une maîtrise médicalisée des dépenses susceptible de concilier les intérêts de l'ensemble des parties concernées.

$\mathrm{Au}$ cours des dernières années, de nombreux travaux de recherche en gestion se sont intéressés aux transformations organisationnelles, professionnelles et en termes d'instrumentation induites par la volonté de développer le «médico-économique » à l'hôpital, à savoir l'intégration de la logique économique dans la logique professionnelle (médicale) dominante. Dans cet article, nous proposons une synthèse des résultats des travaux de recherche appliquée qui ont étudié le déploiement des réformes à l'hôpital et leurs effets. Notre intention première était de comprendre comment ces travaux analysaient la prise en compte de considérations médico-économiques dans les décisions et activités des professionnels hospitaliers.

Réaliser une synthèse de cette littérature est aussi l'occasion de questionner les travaux de recherche eux-mêmes. Quelles sont les questions de recherche étudiées dans ces travaux, les cadres conceptuels mobilisés, les méthodes de recherche mises en œuvre? Une telle analyse permet en creux de faire ressortir quelques-uns des enjeux de la recherche en gestion sur l'hôpital, et de proposer des voies possibles de recherches futures en termes de thématiques comme de modalités.

Dans la suite de cet article, dans une première section nous décrivons tout d'abord de manière plus détaillée, les caractéristiques des réformes, puis le corpus étudié et les méthodes d'analyse que nous avons mises en œuvre. Dans la section qui suit, nous présentons, les résultats de notre analyse de la littérature, que nous avons regroupée en trois catégories de travaux. En effet, nous avons fait le constat que le médico-économique a été étudié par les chercheurs en gestion avec trois perspectives principales, qui correspondent aux trois axes majeurs des réformes : le design organisationnel d'abord avec en particulier la création des pôles, les instruments de gestion ensuite, destinés à suivre, contrôler et piloter la performance des hôpitaux, les acteurs enfin qui mettent en œuvre les réformes et/ou dont la situation et les activités sont touchées par ces dernières. Avant de conclure notre travail, nous discutons dans une dernière section les résultats de nos analyses avec deux clefs d'entrée : ce que les travaux de recherche nous disent, d'une part, de l'intégration de la logique économique dans le monde professionnel hospitalier public en France, et d'autre part, des pratiques de recherche sur ce thème. 


\section{Objet, corpus et méthodes de recherche}

\subsection{Les réformes de l'hôpital public depuis 2005 ou comment intégrer le médical et l'économique}

Les réformes de l'hôpital public en France ont porté à la fois sur le mode de financement comme sur la gouvernance interne et externe des établissements. Dans cette section, nous présentons les caractéristiques principales de ces réformes, en nous concentrant toutefois sur celles qui ont cherché à mieux articuler l'économique et le médical au sein des établissements eux-mêmes, conformément à l'objet de notre étude. Par conséquent, nous n'aborderons pas ici les questions de gouvernance externe, notamment les relations entre les hôpitaux et leurs tutelles - parmi lesquelles on trouve notamment les agences régionales de santé.

La réforme du financement des hôpitaux a porté sur la généralisation rapide, entre 2004 et 2008, du système de tarification à l'activité (dit T2A) pour les activités de Médecine, Chirurgie, Obstétrique (MCO) - soit approximativement 50 \% du budget des hôpitaux. Auparavant, depuis 1983, le financement des hôpitaux publics était entièrement régi par le système dit de la dotation globale. Ce système conduisait à calculer pour l'essentiel le budget de chaque établissement sur la base de celui de la période précédente, revalorisé à partir d'un taux de croissance directeur fixé au niveau national. Ce mode de financement présentait de nombreuses limites : constitution de « rentes de situation » pour les établissements historiquement mieux dotés, faibles incitations à améliorer l'efficience de l'activité ou à la développer, et faible contrôle sur le recours aux soins (Pouvourville, 2009a, 2009b).

La T2A institue, comme dans de nombreux pays européens, un paiement prospectif au cas traité. Ce système, inspiré du mode de paiement déployé pour le système Medicare aux États-Unis, connaît cependant de nombreuses variations dans ses modalités de mises en œuvre (Busse et al., 2011). En France, les tarifs sont définis au niveau national, à partir d'un système complexe de tarification intégrant les coûts observés dans un panel d'établissements représentatifs et des objectifs de gains de productivité. La T2A repose sur une description à granulométrie très fine de l'activité médicale, un processus de codage de l'activité réalisée par les médecins, et un système développé d'information médicale, le PMSI - programme de médicalisation des systèmes d'information pour l'activité MCO (Lenay et Moisdon, 2003; Or et Renaud, 2009; Pouvourville, 2009a). La T2A est donc censée à la fois apporter plus de transparence sur les activités réalisées, et inciter les établissements à améliorer leur efficience. En tant que mode de financement, la T2A, par construction, met en relation activité médicale et performance économique : le total des recettes obtenues par l'établissement sur la base des activités réalisées et des autres financements obtenus peut être comparé à ses coûts globaux afin de mettre en évidence le caractère bénéficiaire ou déficitaire de son fonctionnement. Dans ce système de régulation de l'activité hospitalière, l'objectif de chaque établissement est de parvenir à un fonctionnement équilibrant ses dépenses par ses recettes.

Parallèlement au déploiement de la T2A, la réforme dite de la Nouvelle gouvernance hospitalière a notamment créé au sein des hôpitaux des nouvelles entités organisationnelles, les pôles, eux-mêmes constitués à partir du regroupement de services (Domy et al., 2014). Les pôles ont pour vocation d'améliorer en leur sein la coordination de l'activité de soins entre services, de créer un échelon de responsabilité entre la direction de l'établissement et les services afin de faciliter le management et le pilotage de l'activité, enfin, en confiant la responsabilité des 
pôles à un médecin nommé par le directeur d'établissement, d'organiser le rapprochement entre hiérarchie médicale et hiérarchie administrative dans la gestion de l'établissement.

Dans le même temps, les instances de gouvernance de l'établissement dans son ensemble évoluent : l'ordonnance de 2005 associe pouvoir médical et pouvoir administratif au sein d'une nouvelle instance, le conseil exécutif, regroupant à parité membres de l'équipe de direction et médecins et dont le rôle est notamment de préparer toutes les délibérations du conseil d'administration. La loi dite HPST de 2009 remplace ces deux organes par un directoire et un conseil de surveillance, respectivement. La profession médicale devient majoritaire au sein du directoire, mais les membres en sont nommés par le directeur et ses missions sont réduites par rapport à celles du conseil exécutif qu'il remplace : le directoire devient une instance de concertation pour préparer les décisions du directeur, dont autorité et responsabilités sont renforcées par la loi : par exemple, les médecins chefs de pôles sont nommés dans leurs fonctions par le directeur d'établissement. Le médecin chef de pôle est assisté dans ses fonctions par un cadre supérieur de santé et par un assistant ou responsable administratif, d'où l'appellation de « trio de pôle » associée à la création de ces entités (pour une présentation détaillée de ces réformes, voir par exemple Bérard, 2013b, chapitre V).

La création des pôles et le déploiement de la T2A conduisent à promouvoir une représentation médico-économique de l'activité à travers la mise en œuvre au niveau des pôles, d'un nouvel outil de mesure de la performance, les Comptes de résultat analytiques (CREAs). Tout comme au niveau de l'établissement dans son ensemble, les CREAs mettent en regard les recettes du pôle et ses coûts complets de fonctionnement et permettent donc de faire apparaître le caractère déficitaire ou bénéficiaire d'un pôle, et l'évolution de son résultat d'une année à l'autre. D'autres outils (tableaux de comparaison des coûts de réalisation des activités dits tableaux coûts case mix ou TCCM, tableaux de bord de pilotage) sont destinés à faciliter le dialogue de gestion au sein du pôle en créant « un langage de gestion commun à l'ensemble des acteurs de l'hôpital (guide méthodologique Meah, 2009 : 85) ${ }^{1}$. Ce guide méthodologique recommande, par exemple, que les tableaux de bord de pilotage intègrent des indicateurs d'activité, de ressources humaines, financiers et de qualité des soins.

\subsection{Les travaux sur l'intégration des logiques médicale et économique : construction et description du corpus}

Notre objectif dans cet article étant d'analyser ce que nous apprend la recherche en gestion sur l'intégration des logiques médicale et économique à l'hôpital public suite à ces différentes réformes, nous avons limité notre corpus aux travaux publiés après 2008, donc postérieurement à la mise en œuvre des réformes, et portant uniquement sur l'hôpital public français.

Pour constituer ce corpus, pour des raisons d'accessibilité, nous avons identifié dans un premier temps les principales revues francophones susceptibles de publier des travaux sur l'hôpital. Nous avons donc retenu une revue sectorielle, deux revues publiant des travaux en management public, et six revues généralistes ou thématiques (voir tableau 1).

\footnotetext{
${ }^{1}$ Ces outils sont déployés avec l'aide méthodologique de la Mission nationale d'expertise et d'audit hospitaliers, remplacée à partir de 2009 par l'Agence nationale d'appui à la performance des établissements de santé (ANAP).
} 
Tableau 1 : Liste des revues dépouillées de manière systématique

\begin{tabular}{|l|l|l|}
\hline Revue sectorielle & Revues de management public & Revues en sciences de gestion \\
\hline Journal de Gestion & Politique et Management Public & Comptabilité, contrôle, audit \\
et d'Économie & Revue Française d'Administra- & Finance, Contrôle, Stratégie \\
Médicale & tion Publique & $\begin{array}{l}\text { Gérer et comprendre } \\
\text { Management \& Avenir } \\
\end{array}$ \\
& & $\begin{array}{l}\text { Revue Française de Gestion } \\
\text { Revue Française de Gestion } \\
\text { des Ressources Humaines }\end{array}$ \\
\hline
\end{tabular}

En raison du caractère relativement récent du sujet, susceptible d'expliquer le nombre réduit d'articles publiés, nous avons également cherché à identifier les communications présentées après 2008 à l'occasion de manifestations académiques organisées par des associations thématiques en sciences de gestion, ou avec leur soutien, dont les participants étaient susceptibles d'avoir mené et présenté des recherches sur l'hôpital, et pour lesquels des actes étaient disponibles (voir tableau 2). Nous avons inclus dans notre corpus les travaux présentés et publiés jusqu'en 2014.

Tableau 2 : Actes de manifestations académiques dépouillés de manière systématique

\begin{tabular}{|l|l|l|}
\hline $\begin{array}{l}\text { Manifestations } \\
\text { académiques sectorielles }\end{array}$ & $\begin{array}{l}\text { Manifestations académiques } \\
\text { en management public }\end{array}$ & $\begin{array}{l}\text { Manifestations académiques } \\
\text { thématiques en sciences de gestion }\end{array}$ \\
\hline $\begin{array}{l}\text { Congrès ARAMOS } \\
\begin{array}{l}\text { Colloques Santé Mar- } \\
\text { seille (KEDGE BS, avec le } \\
\text { soutien d'ARAMOS) }\end{array}\end{array}$ & $\begin{array}{l}\text { Colloques de la revue } \\
\text { Politique et Management } \\
\text { Public }\end{array}$ & $\begin{array}{l}\text { Congrès AFC } \\
\text { Congrès AGRH }\end{array}$ \\
\hline
\end{tabular}

Le dépouillement systématique de ces actes et revues a permis d'identifier un premier ensemble de travaux. À partir de la bibliographie de ces derniers, nous avons pu ensuite identifier les travaux publiés par des chercheurs en gestion francophones dans d'autres revues, dans des ouvrages collectifs ou présentés dans des manifestations académiques en France ou à l'étranger et portant eux aussi sur la mise en œuvre des réformes dans les hôpitaux publics français et sur les enjeux médico-économiques de ces réformes. Nous avons choisi de ne pas inclure dans notre corpus les thèses et monographies, en supposant que les recherches correspondantes avaient pour la plupart d'entre elles donné lieu soit à la publication d'articles, soit à la présentation de communications publiées dans des actes de manifestations académiques ${ }^{2}$.

Au total, 67 articles et communications constituent notre corpus. Afin de le structurer, articles et communications ont été répartis en trois catégories : design organisationnel et délégation de responsabilités, instruments de gestion, et acteurs. Comme nous l'avons indiqué plus haut, il s'agit là des trois axes sur lesquels les réformes se sont appuyées et nous avons constaté que pour la grande majorité d'entre elles, les publications étudiées pouvaient être rattachées de manière principale à l'un de ces trois axes (voir également la présentation des méthodes d'analyse ci-dessous).

\footnotetext{
${ }^{2}$ De même, quand une communication a fait l'objet d'une publication dans un ouvrage ou une revue, nous n'avons conservé (et indiqué dans la bibliographie) que les travaux dans ces ouvrages et revues.
} 
Parmi les 67 publications étudiées, 19 portent sur le design organisationnel et la délégation de responsabilités, 30 sur les instruments, et 18 s'intéressent aux acteurs. La liste de ces travaux est fournie en annexe.

De manière plus précise, les travaux s'intéressant au design de l'organisation traitent essentiellement de la délégation de responsabilités managériales au pôle (6 articles), de son appropriation (6), et plus marginalement du trio de pôle (3). Trois autres articles analysent l'évolution de la fonction de contrôle de gestion et de la fonction ressources humaines. Nous avons aussi retenu un article qui présente finement comment les acteurs d'un groupement d'achat sont parvenus à élaborer des indicateurs médico-économiques et un chapitre d'ouvrage qui retrace les principaux questionnements que suscite la mise en place de l'organisation polaire.

Dans les travaux centrés sur les instruments, le thème le plus traité (12 articles) est celui de la mesure de la performance (tableaux de bord, balanced scorecard, CREA). Les autres thèmes sont la contractualisation avec les pôles ( 3 articles), les instruments de gestion sans autre précision (3), l'appropriation des instruments (3), le codage (2) les systèmes d'information (2), le contrôle de gestion RH (1), la communication des résultats (1), le projet d'établissement (1), le PMSI (1) et le budget (1).

Parmi les papiers centrés sur les individus, six s'intéressent exclusivement aux médecins, prioritairement en leur qualité de chefs de pôle, huit aux cadres, trois s'intéressent à une population mixte, un enfin est centré sur le personnel de direction. Notons que le personnel soignant non cadre et les médecins non-chefs de pôle sont peu présents dans ces études ou, le plus souvent, en sont totalement absents.

\subsection{Analyser les travaux de recherche : méthodes mises en œuvre}

Nous avons tout d'abord effectué un dépouillement systématique des revues et actes constituant notre corpus.

Dans un premier temps, nous avons présélectionné l'ensemble des articles et communications traitant de l'une des réformes mises en œuvre et retenu également ceux qui, moins centrés sur les réformes, traitent néanmoins de la relation ou de la tension entre « le médical » et « l'économique » dans un contexte hospitalier public postérieur à la mise en œuvre de la Nouvelle gouvernance hospitalière.

Pour chacun des articles présélectionnés, nous avons en outre cherché à identifier la principale thématique de la recherche, de manière à la rattacher à l'un des trois axes que nous avons retenus : design organisationnel, instruments de gestion, et acteurs.

Pour analyser chacun de ces articles, nous avons élaboré une grille précisant, de manière plus détaillée, le thème de l'article, la question de recherche étudiée, le cadre conceptuel mobilisé, le design et les méthodes de recherche, et les principaux résultats de la recherche. Une dernière catégorie de notre grille était destinée à évaluer, de manière plus subjective, la contribution de la recherche sur la base de l'importance, de la qualité ou de l'originalité des résultats obtenus ${ }^{3}$.

Afin de tester la pertinence des catégories d'analyse retenues pour élaborer la grille et d'homogénéiser la manière de les comprendre et de les mobiliser, chacun des co-auteurs

\footnotetext{
${ }^{3}$ Ces différents éléments sont rappelés dans chacun des trois axes d'analyse dans la section 2 suivante, et en particulier les cadres théoriques mobilisés par les auteurs dont nous avons analysé les travaux.
} 
a lu et analysé séparément trois à cinq articles. Nous avons ensuite croisé nos analyses à l'occasion d'une réunion qui a permis de discuter des choix effectués et de s'accorder sur des procédés d'analyse homogènes.

À la suite de cette étape d'ajustement, et en fonction des domaines de compétences respectifs des co-auteurs de ce travail, nous avons pris en charge l'analyse des articles de l'un des trois axes du corpus, à l'exception des articles sur les instruments de gestion, plus nombreux, pour lesquels le travail d'analyse a été réparti entre deux des chercheurs.

Plusieurs réunions, à différentes étapes de l'analyse du corpus, ont permis de continuer à vérifier que la grille d'analyse était mise en œuvre de manière homogène. Ces réunions ont aussi permis de valider des décisions d'exclusion du corpus lorsqu'il apparaissait, après lecture approfondie, que les articles n'abordaient que de manière marginale les enjeux médico-économiques, ou encore qu'ils ne relevaient pas d'articles de recherche mais de points de vue d'experts ou de témoignages de bonnes pratiques.

Afin de pouvoir dénombrer et analyser de manière structurée notre corpus, nous avons choisi de rattacher chaque article à un seul des trois axes retenus : design/instruments/acteurs. Les réunions de l'équipe de recherche ont donc aussi permis de prendre les décisions de classement des articles dans l'une des trois catégories pour ceux d'entre eux présentant par le thème étudié plusieurs rattachements possibles.

Dans la section qui suit, nous présentons les résultats de nos analyses selon ces trois entrées thématiques.

\section{L'état des connaissances sur l'intégration des logiques médicale et économique selon trois entrées thématiques}

Les trois entrées sont : le design organisationnel (et la délégation de responsabilités), les outils de gestion et les individus. La méthode choisie dans les papiers étudiés est presque exclusivement l'étude de cas, très souvent unique. Plusieurs d'entre eux mentionnent leur caractère « exploratoire ». Il y a très peu de citations croisées des travaux identifiés laissant penser que les recherches sont peu cumulatives. Parmi ces travaux, un petit nombre est issu de recherche intervention (7). Les papiers reposant sur une enquête par questionnaires sont marginaux (4 au total). Notons que quelques papiers reposent sur l'expertise des auteurs et qu'un seul papier utilise des données secondaires (celles du rapport de la Mission cadres hospitaliers - voir Singly, 2009).

\subsection{Reconfigurer pour mieux intégrer : le levier des pôles}

19 articles questionnent les conditions et les modalités d'une meilleure articulation entre la sphère médicale et la sphère économique à travers la réorganisation hospitalière. 15 de ces articles s'intéressent à la nouvelle organisation polaire (délégation, appropriation et trio de pôle) tandis que trois articles (Crozet et al. 2008; Dos Santos et al. 2014; Lartigau, 2009) étudient les conséquences sur la fonction de contrôle de gestion et ressources humaines. C'est très souvent par le prisme du contrat de pôle ou des outils de gestion (CREA, tableaux de bord...) mis à disposition des pôles qu'est abordée la restructuration, paraissant indiquer que cette réforme est avant tout une question d'outillage gestionnaire. 
Les auteurs mobilisent très majoritairement le modèle de la bureaucratie professionnelle pour caractériser l'hôpital, à l'exception de Vallejo et al. (2014) qui le considèrent comme une configuration divisionnelle, Angelé-Halgand (2014) comme un ensemble de contrats entre agents et principal, et Moisdon (2008) comme l'imbrication des quatre mondes professionnels de Glouberman et Mintzberg (2002). Un article fait référence à l'approche néo-institutionnelle pour rendre compte des pressions isomorphes pesant sur un hôpital (Grenier et Bernardini, 2013). Force est de constater que, dans un peu moins de la moitié des articles retenus, les cadres théoriques sont donnés sans pour autant soutenir l'analyse des situations étudiées, qui prend alors plutôt la forme d'une description empirique.

Le message délivré par les articles est très souvent le même : la mise en place des pôles est difficile, ils sont peu ou pas une entité de prise de décision plus déconcentrée, même s'ils s'installent progressivement comme structure reconnue.

Un seul article questionne véritablement la pertinence des découpages polaires au sein de l'hôpital étudié (Gouffé et Cargnello-Charles, 2014).

La première difficulté abondamment relatée par les articles, est la résistance des directions fonctionnelles à donner aux pôles une pleine responsabilité managériale. Tout se passe comme si, en dépit d'un principe de délégation, les directions de l'information médicale, les directions des ressources humaines, ou les services de contrôle de gestion voyaient leur rôle renforcé dans un contexte marqué par la pénurie de ressources financières et la mise en œuvre d'une tarification à l'activité véhiculant une vision économique des décisions d'actes de soin (Lartigau, 2009). C'est un manque de confiance dans les pôles, et donc en particulier dans les médecins, à être des gestionnaires responsables de leur activité qui expliquerait la difficile délégation (Bérard, 2013a; Gavault et al., 2014). Les pôles disent alors ne pas pouvoir jouer pleinement leur rôle par manque d'information (Guicheteau et Maestre-Levèvre, 2009), par méconnaissance des choix stratégiques de l'établissement (Angelé-Halgand, 2012), par manque de compétences pour décider sur la base d'indicateurs médico-économiques et finalement par difficulté à comprendre ce que signifie « délégation » (Bérard, 2013a; Dos Santos et al., 2014). Deux articles montrent en miroir la difficulté d'une DRH (Crozet et al., 2008) ou d'une direction contrôle de gestion (Dos Santos et al., 2014) à appréhender la manière dont leur rôle peut évoluer dans la nouvelle configuration polaire.

Le deuxième écueil à la mise en place de pôles comme lieux privilégiés de la fabrication du médico-économique relève des outils pourtant supposés la faciliter. L'incomplétude de certains outils (Guicheteau et Maestre-Levèvre, 2009, cas des CREA), ou au contraire l'abondance d'indicateurs (Gouffé et Cargnello-Charles, 2014), l'opacité de leur construction, ne permettent pas aux pôles d'exercer les responsabilités qui leur sont normalement dévolues.

Le pôle devient le réceptacle d'un contrôle purement économique de ses activités (Bérard, 2013a; Vallejo et al., 2014) sans que sa mise en place n'aboutisse au travail de re-conception des méthodes de production du soin que l'on aurait pu en attendre (Gavault et al., 2014; Moisdon, 2008). Un seul article relate comment un trio de pôle cherche à défendre une logique médico-économique pour contrer le poids grandissant du raisonnement économique (Grenier et Bernardini, 2014).

Malgré ces difficultés, « l'histoire est en construction » (Havard, 2014) et des travaux mettent l'accent sur la constitution progressive des pôles qui acquièrent une certaine visibilité (Flachère, 2014). Cette histoire en marche est facilitée, là où le découpage des pôles a été fait en cohérence avec les orientations stratégiques de l'hôpital (Nobre et Lambert, 2012), 
quand les contrats de pôle sont réellement négociés entre DG, directions fonctionnelles et chef de pôles (Angelé-Halgand, 2012), grâce à la déviance de certains comportements (Gouffé et Cargnello-Charles, 2014) ou lorsque des rencontres facilitent l'émergence de représentations communes et d'apprentissages (Guicheteau et Maestre-Levèvre, 2009, Gouffé et Cargnello-Charles, 2014; Vallejo et al., 2014).

\subsection{Les instruments de gestion : le rendez-vous manqué avec une logique médico-économique}

Les instruments de gestion sont au cœur de l'intégration du médical et de l'économique pour plusieurs raisons. Certains de ces instruments, comme les CREAs visent directement à introduire la dimension économique au niveau de la responsabilité médicale. Ils s'appuient directement sur la T2A, qui repose elle-même sur une représentation médico-économique de l'activité - celle des Groupes homogènes de malades. D'autres instruments, comme les tableaux de bord, ont pour vocation de capturer les différentes dimensions de la performance en particulier économique et médicale. Pour autant, la manière dont sont élaborés les critères médico-économiques n'est que rarement centrale dans les articles étudiés. En effet, il ressort de ces articles que de nombreuses questions se posent avant même d'étudier l'impact de l'introduction d'instruments sur l'articulation des logiques médicales et économiques : comment construire un tableau de bord ? Comment les instruments sont-ils utilisés ? Comment sont-ils perçus et appropriés? Lorsque leur rôle dans la gestion du médico-économique est abordé, c'est pour mettre en évidence un effet beaucoup plus indirect et symbolique (Bonnier, Flachère et al. 2013; Crémieux et al., 2013) : dans les travaux analysés, il n’y a pas d'exemple d'instruments directement mobilisés pour une prise de décision appuyée sur des données faisant le lien entre le médical et l'économique et leur analyse.

Sur les 30 papiers sélectionnés, cinq articles ont une contribution à la fois empirique et théorique. Ces articles mobilisent les concepts ou cadres théoriques suivants : objet-frontière, RAPM (reliance on accounting performance measures), conflit de rôle, théorie de l'acteur-réseau et socio-matérialité. La contribution des autres articles est empirique. Une vingtaine d'articles mobilisent des cadres théoriques ou conceptuels avec une visée interprétative. Ainsi, les articles sur l'appropriation reposent sur les théories de l'appropriation des instruments de gestion, un article s'appuie sur la théorie C-K (concepts - connaissances, Hatchuel, 2002), un sur la théorie de la structuration, un autre sur l'approche téléologique des systèmes d'information, un dernier enfin sur le concept de contrôlabilité. Si le NPM (New Public Management), la distinction entre organisation bureaucratique et organisation professionnelle, le concept de découplage ou celui d'accountability sont évoqués, ils ne sont pas utilisés dans les analyses. Soulignons enfin qu'un nombre significatif d'articles (10) ne repose sur aucun cadre théorique ou analytique.

D'un point de vue empirique, ces articles montrent que les instruments peuvent être utilisés de manières très différentes selon les contextes. Ils conduisent donc à souligner l'importance d'adopter une approche organisationnelle et contextualisée, et non comportementale uniquement, pour étudier l'introduction de nouveaux instruments à l'hôpital (Georgescu et Naro, 2012). Ainsi, les CREA peuvent être utilisés comme support à des incitations monétaires ou non, les modalités de ces incitations pouvant être très variées, ils peuvent être produits trimestriellement, semestriellement ou annuellement et analysés dans des réunions impliquant tous les pôles ou un seul pôle, etc., (Bérard et al. 2013; Bonnier, 
Flachère et al. 2013 ; Flachère, 2014). Par ailleurs, la façon dont les charges indirectes sont allouées aux pôles et dont les prix de transfert sont calculés est différente selon les contextes et plus ou moins transparente. Ces modes d'utilisation peuvent être analysés comme hétérodoxes, néanmoins intéressants, comme dans l'analyse de la contractualisation faite par Bérard et Steyer (2013).

Ces articles mettent aussi en évidence de nombreux cas d'appropriation des instruments par les professionnels alors que la littérature internationale a plutôt documenté des cas de résistance en particulier à la fin des années 1990 (Jacobs, 1995; Jones et Dewing, 1997 ; Doolin, 1999; Lowe et Doolin, 1999). Toutefois, la mobilisation des instruments est sans lien avec leur impact sur la performance. Ainsi, dans un cas, l'introduction d'un tableau de bord a comme principal effet de rendre possible la tenue de réunions entre managers et médecins au cours desquelles des questions médico-économiques sont discutées, mais sans que le tableau de bord soit réellement mobilisé dans ces discussions (Bonnier et al. 2013). Il semble donc important de considérer les instruments comme pouvant avoir des apports variés ne se limitant pas à l'amélioration de la performance. Bérard et Steyer (2013) précisent ainsi que l'intérêt de l'instrumentalisation n'est pas nécessairement à rechercher dans son impact fonctionnel direct, mais dans sa capacité à permettre aux acteurs de gérer l'ambiguïté.

Ces observations ne sont pas nécessairement surprenantes si l'on considère que les instruments doivent être transformés par les acteurs pour être appropriés (Giraud et al., 2011). Mais cette appropriation ne semble pas maîtrisée : les cas relatés mettent plutôt en évidence une absence de réflexion sur les objectifs des instruments par les acteurs qui les mettent en œuvre localement. Or s'approprier les outils ne signifie pas uniquement les transformer pour les adapter au contexte local. Il s'agit aussi de respecter «l'intention» que les outils sont supposés servir.

La diffusion à grande échelle d'instruments standards dans l'ensemble des établissements à partir du niveau national semble reposer sur l'idée que ces instruments sont des vecteurs de conformation des pratiques dont l'effet sur la performance est univoque et ne nécessite ni de prendre en compte les caractéristiques du contexte de déploiement, ni d'expliquer les mécanismes par lesquels cet effet est supposé advenir. Les résultats empiriques indiquent au contraire que le contexte est essentiel puisque les instruments sont transformés localement et que les effets sont très variés. Ils sont renforcés par des articles qui bien que n'apportant pas de contribution empirique présentent des cadres conceptuels montrant par exemple l'importance des éléments informels comme la communication sur les effets des instruments.

Un autre résultat qui ressort de ces articles concerne les différentes finalités des instruments, en particulier l'évaluation et l'apprentissage. Il est alors important de bien articuler la mise en œuvre des instruments avec ces finalités. Ainsi, Moisdon (2012) explique le peu de changements à l'hôpital malgré les nombreuses réformes par le fait que les outils sont conçus avec une finalité d'évaluation et d'incitation, dans le but d'éviter au régulateur de se mêler de l'organisation. Ils n'aident donc pas les acteurs internes à se guider dans une réelle reconfiguration de leur organisation. D'autres articles montrent en quoi les difficultés rencontrées dans la mise en œuvre des instruments sont liées à l'inadéquation entre leurs caractéristiques techniques (leur design) et les usages qui en sont faits (Crémieux et al., 2013). Là aussi, ces résultats sont appuyés par des articles qui, bien que n'apportant pas de contribution empirique, présentent des cadres conceptuels pour permettre de réfléchir 
aux changements en cours, dans le but d'ouvrir à des conceptions plus riches du contrôle de gestion et des instruments, et notamment de ne pas les cantonner à une fonction d'évaluation. Ces résultats plaident donc pour réfléchir aux objectifs des instruments et à leur apport et à ne pas penser les outils en termes de conformation. En particulier, ils devraient conduire à penser la diffusion comme des expérimentations dans une logique d'apprentissage sur les instruments eux-mêmes (Moisdon, 2008) et non comme l'application d'un outil standard. Or, si les articles mettent bien en évidence des expérimentations locales, ils montrent aussi que ces expérimentations ne font que peu l'objet d'évaluation et que dans ce cas l'évaluation reste locale.

D'autres observations complètent ces résultats : Les contrats de pôles ont d'autant plus de chances d'améliorer les prises en charges des patients, que la contractualisation se fait sur des projets plutôt que sur des objectifs de résultat (Kauffmann, 2008); la mise en œuvre des instruments au sein de pôles est problématique en raison d'incohérences dans les choix de regroupement de services au sein des pôles (Margarit et Peyret, 2008); la mesure de la performance est rendue difficile par la multiplicité des parties prenantes (Montalan et Vincent, 2011) ou par la complexité de l'organisation (Arthus et al. 2009; Nobre et Haouet, 2011); le cloisonnement soignant/médecin peut être préjudiciable à la mise en œuvre de certains instruments (Dumas et al. 2012).

Enfin, certains articles ne se placent pas au niveau micro-organisationnel, mais au niveau institutionnel et ne reposent pas sur une base empirique mais analytique seulement. Ils discutent de la cohérence globale des réformes - incohérence entre régulation confiée aux ARS et concurrence soutenue par la T2A (Pascal et al., 2011); pertinence d'une évaluation de la performance qui ne prend pas assez en compte le point de vue du patient et du personnel (Le Pogam et al., 2009) ou de leurs intentions - les outils ne viseraient pas à l'amélioration de la performance, mais à la mise en place d'un gouvernement à distance (Angelé-Halgand et Garrot, 2014).

\subsection{Des professionnels aux prises avec la gestion}

Si tous les papiers s'intéressant aux acteurs mentionnent les tensions entre les logiques économiques et médicales, la question de la combinaison de ces logiques est rarement le sujet central. Les travaux consistent le plus souvent à regarder comment les professionnels font face et mettent en œuvre des éléments de la réforme, qu'il s'agisse d'un rôle, d'outils de contrôle de gestion ou plus largement de la pression accrue sur les ressources. Certains (6) dépassent la stricte étude de la mise en œuvre pour s'intéresser aux conséquences de ces réformes sur les compétences, l'identité, l'implication ou le stress. Lorsque l'articulation du médicale et de l'économique est abordée, c'est à travers les enjeux d'appropriation des logiques et pratiques de gestion par les professionnels, dans un contexte caractérisé par l'exercice de contraintes nouvelles. La notion d'hybridation pour rendre compte de ce contexte d'action appelé à se complexifier est utilisée dans certaines études (3), mais est peu ou pas définie.

Certains travaux partagent un même objet d'étude. Les résultats sont toutefois contrastés, voire contradictoires. Cinq s'intéressent à l'évolution du travail des cadres de santé. L'activité de gestion formalisée, élément important de la managérialisation de l'hôpital (gestion de projet, information des tableaux de bord) est considérée par Detchessahar et 
Grevin (2009) comme occupant un temps croissant, ne laissant pas de place pour «penser le travail ». Coulon (2011) au contraire souligne que l'activité de résolution de problème à court terme reste l'activité principale, reléguant l'activité de gestion formalisée au second plan du travail et des préoccupations des cadres. Plus précisément, des compétences nouvelles autour de la capacité à organiser les flux des biens et personnes seraient stimulées même si les compétences traditionnelles de soins restent nécessaires à la légitimité des acteurs du soin (Dumas et Ruiller, 2013). Bouret (2008) met en avant que, si les cadres ont bien des activités de conception, consistant à «penser le travail », c'est en temps caché. C'est un travail « invisible ». Trois papiers s'intéressent à l'identité médicale. L'injonction à faire de la gestion provoquerait une fragilisation identitaire (Pouvourville, 2010) ou au contraire un renforcement de l'identité professionnelle (Georgescu et Rivière, 2013), ou encore toucherait peu à l'identité des médecins (Valette et Burellier, 2014).

Les autres travaux traitent d'objets variés et les résultats sont épars. Citons en quelquesuns pêle-mêle. Chez les cadres, la nécessité de mettre en œuvre les réformes fait émerger une entité collective qui reste toutefois hétérogène (Divay et Gadea, 2008). Concernant les médecins, le mentorat et les relations inter-génération seraient un facteur favorable à l'hybridation de leur rôle (Duchamp et Persson, 2012). Par ailleurs, si beaucoup de chefs de pôles s'adaptent tant bien que mal à leurs nouveaux rôles prescrits, certains d'entre eux conservent un rôle traditionnel et «contraignent ainsi l'organisation » à s'adapter à des conceptions individuelles de ce qu'est un chef de pôle (Valette et al., 2011). Fiore et Sampieri-Teissier (2011) mettent plus simplement en avant la faible autonomie des chefs de pôles en matière de contrôle de gestion. L'implication des médecins est, elle, affectée différemment par la plus grande pression budgétaire : lorsque cette pression est exercée par l'administration, on observe alors une baisse de l'implication affective, lorsqu'elle l'est par les médecins, on observe une augmentation de l'implication calculée (Georgescu et Commeiras, 2012).

Comme dans les papiers examinés dans les parties précédentes, il y a parfois besoin de caractériser le contexte organisationnel dans lequel évoluent les acteurs. Le modèle de Glouberman et Mintzberg (2002) qui décrit l'hôpital à travers quatre mondes, celui du « cure », du «care », du contrôle, et de la communauté est évoqué deux fois. La pluralité des logiques et la nécessité de trouver des arrangements pour les faire cohabiter sont une caractéristique structurelle de l'hôpital, plus qu'une conséquence de réformes nouvelles. Valette et Burellier (2014) suggèrent toutefois que la spécificité de la période en cours est la délégation explicite aux médecins des recherches d'arrangements entre soins et gestion, entre le « cure », le « care » et le « contrôle »; mais Detchessahar et Grevin (2009) rappellent que c'est le cas depuis toujours pour les cadres de santé.

Les cadres d'analyse mobilisés sont principalement issus de deux types d'approches, les tensions de rôle ( 6 papiers) et la psycho-sociologie du travail ( 3 papiers). Les premières mobilisent les notions de tensions de rôles (Kahn et al., 1964), de gestion des contradictions (Lewis, 2000), de stratégies de coping (Lazarus et Folkman, 1984), d'ajustement entre rôles requis et rôles joués (Nicholson, 1984) avec ses conséquences éventuelles sur l'implication et l'identité professionnelle. Elles ont en commun de faire l'hypothèse que les différentes logiques créent des tensions, voire des contradictions et que les individus vont devoir déployer des stratégies pour y faire face. Ces tensions peuvent être caractérisées par leur « localisation ». Rivière et al. (2013) identifient ainsi chez les cadres des tensions 
inter-émetteurs, intra-émetteur, inter-rôles, intra-personnelles qui donnent lieu à diverses stratégies d'ajustements. Elles peuvent être caractérisées par leur origine. Valette et al. (2011) ou Valette et Burellier (2014) pointent pour les chefs de pôle, des tensions liées aux différentes définitions de la performance, aux différentes formes de loyauté, aux problèmes d'organisation du temps et du travail et aux valeurs. Les approches en pyscho-sociologie du travail regardent, elles, comment s'articulent le travail professionnel et le travail de gestion, en privilégiant la dimension collective de ce travail. Cette articulation renvoie selon les auteurs à une situation de domination ou, au contraire, est analysée comme un espace de ressources pour l'action.

Les travaux sur les acteurs apparaissent ainsi dispersés et lorsqu'ils abordent des thématiques identiques, les résultats sont contrastés voire contradictoires. L'absence d'accumulation des connaissances ne permet pas de discuter ces différences. Les approches par les tensions de rôle et la gestion des contradictions et paradoxes sont les seuls cadres théoriques réellement identifiés dans ces travaux pour rendre compte de l'effet sur les individus de l'ambition de construire une régulation plus médico-économique.

\section{Discussion : des travaux et des résultats encore en devenir}

Cette revue de littérature met en lumière un certain nombre d'éléments portant à la fois sur la question de l'intégration médicale et de l'économique à l'hôpital mais aussi sur la conduite des recherches en gestion dans le champ.

\subsection{Ce que l'on apprend sur l'intégration des logiques médicale et économique}

\section{Le médico-économique : un contexte, peu de résultats analysés}

Notre premier résultat est que la question de l'intégration du médical et de l'économique n'est pas traitée en tant que telle dans les recherches. Étudier cette question nécessiterait, par exemple, d'observer comment des pratiques intègrent des éléments des logiques ou mondes professionnel et économique, comment les capacités de décisions individuelles et collectives sont modifiées pour permettre de prendre en compte des informations économiques et médicales, en quoi des solutions nouvelles sont proposées pour résoudre des tensions anciennes.

Or : a) les travaux centrés sur les individus, s'interrogent sur ce que produit la nouvelle logique de gestion sur les professionnels mais s'intéressent peu à la manière dont ces professionnels par leurs actions, articulent ou pas des logiques économiques et professionnelles ;

b) les recherches centrées sur le design de l'organisation soulignent la difficulté à mettre en pratique la délégation de gestion et la responsabilisation des pôles mais la régulation « au concret » n'est pas restituée. Rien n'est dit par exemple sur la manière dont sont conçus les projets et se déroulent les arbitrages. Il est alors difficile d'identifier ce que les pôles transforment ou pas dans la gouvernance d'ensemble; et finalement;

c) les recherches centrées sur les instruments ne restituent pas la manière dont les informations conditionnent et transforment ou pas les prises de décision.

Autrement dit, les recherches portent sur la mise en place des outils ou structures portées par les réformes, sur les difficultés de cette mise en place, sur leurs conséquences sur les individus, mais pas sur leurs conséquences sur les pratiques médico-économiques qui 
sont pourtant une des finalités de ces réformes. C'est pourquoi nous considérons que, dans notre corpus, la question de l'intégration du médical et de l'économique n'est pas un objet de recherche en tant que tel mais semble plutôt une donnée ou un élément de contexte par rapport au véritable objet des travaux étudiés.

Comment expliquer ce résultat? Plusieurs explications, non exclusives, sont possibles. Tout d'abord, la question de l'intégration du médical et de l'économique dans les pratiques des acteurs peut être prématurée à la date où ont été rédigés les papiers retenus. Il s'agit encore avant tout de comprendre comment la réforme est déployée et se met en œuvre, plus que d'analyser ses conséquences sur les processus de décision et d'action. Une autre raison possible est que les transformations ne touchent de fait pas ou peu aux pratiques. L'organisation serait alors « hypocrite » (Brunsson, 1992) développant beaucoup d'énergie pour mettre en œuvre les nouveaux dispositifs formels de l'organisation mais protégeant le cœur des pratiques établies avec tout autant d'énergie. Une dernière raison pourrait être que les organisations se transforment mais selon un mode qui exige des dispositifs méthodologiques pour les observer, et théoriques pour les comprendre, qui sont spécifiques. En particulier, la diversité des pratiques, des effets et des finalités des instruments que nous avons mise en évidence plaiderait pour une observation plus fine des pratiques qu'elle ne l'est en générale faite, ce qui rejoint la recommandation de Pépin et Moisdon (2010).

\section{Un cadre théorique encore embryonnaire}

Étudier la façon dont les dimensions médicales et économiques s'articulent dans les représentations et dans les pratiques passe par des cadres analytiques et conceptuels qui restent à encore eux-mêmes à construire en partie. En ce qui concerne les instruments de gestion, l'appareillage théorique devrait notamment développer un meilleur équilibre entre les dimensions techniques et psycho-sociales des instruments, en réinvestissant la première (Baldvinsdottir et al. 2010). Pour aller dans ce sens, les approches du courant socio-technique pourraient s'avérer prometteuses (Aggeri et Labatut, 2010). Bérard et al. (2015) proposent ainsi un cadre d'analyse du déploiement d'outils de gestion qui articule une analyse des outils de gestion eux-mêmes fondés sur la grille proposée par Hatchuel et Weil (1992) - substrat technique, philosophie gestionnaire, théorie de l'action - et une analyse du contexte organisationnel selon deux axes : les acteurs impliqués dans la dynamique d'action et les conditions de gouvernance. Pour étudier les instruments, il faut aussi développer une « grammaire instrumentale » qui, selon Moisdon (2008), reste trop embryonnaire.

La dimension «pluraliste » (Denis et al., 2007) mettant en avant la confrontation à l'hôpital des logiques différentes, voire contradictoires semble incontournable. Elle est prolongée dans certains travaux par une réflexion sur les hybridations (Kurunmäki, 2004) de compétences, de rôles, d'identité, d'instrumentation, notion prometteuse pour aller au-delà de la caractérisation structurelle de l'organisation hospitalière ou au-delà du constat des tensions existantes entre les mondes. Ainsi, dans la littérature, la confrontation entre logiques est analysée dans deux directions : en analysant les tensions qui exposent l'organisation à une dérive d'activité, une perte d'objectifs ou à des pratiques de déviance (Battilana et Dorado, 2010, Mazouz et al., 2012), en considérant la manière dont le pluralisme prend forme dans l'organisation : le refus de la nouvelle logique (Kraatz et Block, 2008), le remplacement de la logique initiale (Reay et Hinings, 2009), ou encore l'hybridation des 
logiques. Parler d'hybridation suppose que les tensions entre logiques concurrentes peuvent se résoudre dans l'émergence d'une logique hybridée (Battilana et Dorado, 2010; Grenier et Bernardini, 2016).

Prendre à bras-le-corps l'enjeu d'hybridation nécessite de regarder comment s'articulent ces logiques et de suivre les « arrangements » auxquels elles donnent lieu. Dans quelle mesure, les systèmes d'informations, les organes de décisions, les organisations formelles, les dispositifs de contrôle, les compétences, les identités, les prises de décision cohabitent, dominent, se segmentent, se transforment, se combinent?

\section{La variété des pratiques d'établissement}

Un troisième constat est que les recherches rendent compte d'une grande variété dans les pratiques d'établissements. Par exemple, les comptes d'exploitation de pôles ou «CREA » sont construits et mobilisés de façon très différente d'un établissement à l'autre, les modes de formations des pôles, les délégations associées, les profils des chefs de pôle et des équipes de direction des pôles sont eux aussi différents d'un établissement à l'autre. Cette variété peut être expliquée par le temps de la réforme. Les établissements tâtonnent, essaient, font des choix en fonction de leurs contraintes ou leurs priorités. Toutefois, elle s'inscrit dans un contexte très normé dans lequel la tutelle des établissements cherche à s'appuyer sur des dispositifs homogènes à la fois en termes de structure et d'instruments pour mettre en œuvre les transformations jugées pertinentes et lutter alors contre les disparités. La variété est alors le signe que les dispositifs font l'objet d'un processus d'appropriation qui va au-delà de la simple adaptation à un contexte particulier. L'usage de nouveaux dispositifs conduit les personnes à apprendre comment les utiliser et quels sont leurs effets à la fois contraignants et habilitants, et par là même à les transformer. Si cette interprétation est juste, elle conduit à s'interroger sur les modalités de déploiement des politiques publiques et à considérer l'introduction de nouvelles organisations ou de nouveaux instruments comme des processus d'expérimentation plutôt que comme la diffusion de bonnes pratiques ou de solutions conçues de façon centralisée. Cela nécessite des allers-retours entre les niveaux macro et micro-organisationnels auxquels la recherche pourrait contribuer.

\subsection{Ce que l'on apprend sur les pratiques de recherche}

L'analyse conduite sur les articles retenus permet d'aller au-delà de la présentation de ce qu'on apprend sur les transformations en cours. Elle donne à voir les recherches pratiquées en France, en sciences de gestion sur le secteur hospitalier. Nous nous autorisons donc ici un regard critique sur nos pratiques.

\section{Des recherches « isolées »}

Nous avons mentionné que l'étude de cas unique, souvent présentée comme exploratoire, est largement privilégiée. Les recherches existantes sont par ailleurs peu citées, souvent parce que les problématisations sont singulières, liées à la demande du terrain. Si la méthode des cas permet d'aller au cœur des pratiques des établissements et est rendue possible par des relations souvent étroites entre une équipe de chercheurs et les établissements, nous pensons qu'elle ne se suffit pas à elle-même. Elle exige accumulation et confrontation qui sont insuffisamment pratiquées. Accumulation pour repérer les invariants et les spécificités 
des résultats obtenus, confrontation pour mettre à l'épreuve l'interprétation des chercheurs . L'accumulation peut passer par la réplication des recherches et la référence aux recherches existantes. La confrontation exige des espaces de discussions des résultats. Le développement d'associations académiques spécialisées telles qu'ARAMOS (Association pour la recherche appliquée sur le management des organisations de santé) et les manifestations associées (congrès ARAMOS, Colloque Santé organisé à Marseille) constituent de nouveaux espaces pour structurer de manière très utile la recherche. De même, l'initiative de la revue Gérer et Comprendre qui, suite à l'article de Valette et Burellier (2014) publie un commentaire de Kletz (2014) invitant à relativiser les résultats, peut être soulignée. Alors que les auteurs de l'article mettent en avant le poids du «bricolage » individuel des chefs de pôle dans la fabrication de leur nouveau rôle, F. Kletz choisit quant à lui de mettre l'accent sur les initiatives des établissements pour accompagner ces changements.

Par ailleurs, comme nous l'avons vu, ces cas singuliers conduisent à mettre en évidence une grande variété de pratiques, qui pose la question du dialogue entre les chercheurs et les acteurs du champ. S'agit-il pour le chercheur de détecter parmi cette variété, les bonnes pratiques afin de favoriser leurs duplications et/ou l'élaboration d'un cadre d'action harmonisé par le ministère et ses agences? Faut-il au contraire souligner la dimension micro-sociale des réformes, implantées dans des organisations qui ont une histoire, des acteurs, des enjeux de pilotage, et des contraintes qui agissent significativement sur leur contenu? La variété est alors irréductible parce qu'elle est le produit de processus singuliers d'appropriation des instruments, des rôles, des structures par les individus et les organisations dont le chercheur doit témoigner. Ou faut-il tenter de faire sens de ces pratiques en mobilisant les cadres conceptuels adaptés pour permettre aux acteurs du champ de bien formuler leurs questions et d'avancer dans la conception de réponses innovantes à ces questions?

\section{Les absences}

Une lecture en creux des travaux sélectionnés, permet aussi de souligner ce qu'on ne trouve pas dans les recherches françaises et qui nous apparaît « manquant ». Tout d'abord, des thèmes, importants au regard des transformations en cours ou des enjeux des réformes sont absents. Il en est ainsi de la coordination du travail, de la gestion de la transversalité, enjeu médicoéconomique important, à l'origine de la création des pôles. Nous n'avons pas trouvé non plus de travaux rendant compte de processus de conduite de projets médicaux dont on peut se demander comment ils intègrent les dimensions économiques et les nouvelles relations entre pôles, et entre pôles et directions fonctionnelles ou direction générale. L'implantation du lean management, en cours dans de nombreux hôpitaux, dont l'objectif est d'améliorer les processus en optimisant les ressources et la gestion la qualité des prises en charge, semble très peu étudiée dans les travaux de recherche en gestion sur l'hôpital en France. De même, l'innovation médicale ou dans le soin n'est pas traitée en lien avec les enjeux médico-économiques

Enfin, il y a peu de références aux travaux étrangers, comme si la mise en débat au niveau international n'était pas possible au regard des spécificités françaises.

\section{Les contributions et la question de la recherche appliquée}

Les approches nous semblent par ailleurs assez peu outillées théoriquement. Les cadres d'analyse sont relativement limités. On note aussi des fragilités méthodologiques. En particulier, il est surprenant de donner un statut académique à des articles écrits par des praticiens 
sur la base de leur expérience, qui ne mobilisent pas de design de recherche solide. De même, plusieurs articles reposent sur des données insuffisantes, par exemple sur trois entretiens. Les articles se réclamant d'une méthodologie de type interventionniste sont souvent le récit d'une démarche de conseil sans réelle mise en œuvre des principes méthodologiques de la recherche intervention ou de la recherche action. Enfin, plusieurs articles manquent d'un questionnement théorique ou empirique clair et justifié par une revue de littérature. Globalement, la contribution d'une proportion significative d'articles reste donc limitée.

Dumez (2010) rappelle qu'une bonne recherche qualitative repose sur la qualité du problème posé, problème construit comme une tension entre un état du savoir et un nonsavoir et la mise en place d'un cadre théorique qui s'expose à la critique. Le problème posé intègre donc de manière indissociable de l'empirisme et un cadre analytique. Le savoir et non savoir sont construits différemment selon la nature de la recherche. S'il y a un enjeu dans des recherches fondamentales à construire l'état des savoirs et non savoirs dans l'espace de la théorie, alors que les données participent, elles, à la résolution des tensions, la recherche appliquée disciplinaire construit l'état du savoir et non savoir de manière empirique, au plus près des pratiques. La mise en tension avec le cadre théorique exige alors une liberté dans le choix et la construction d'un cadre analytique approprié pour résoudre la tension.

La recherche appliquée doit faire face à trois défis. Le premier est de faire la part entre le savoir et le non-savoir dans le champ. Ceci relève à la fois de la responsabilité du chercheur, qui doit se réapproprier l'état des connaissances empiriques, et des acteurs du champ qui doivent rendre ce savoir disponible. La deuxième est de mobiliser des cadres théoriques producteurs de sens. On peut à cette occasion se demander si des phénomènes de « modes théoriques » ne sont pas un frein à la production de connaissances appliquées. Il y a ici un enjeu pour des revues disciplinaires à « autoriser » la mobilisation de grilles d'analyse qui, bien que « désuètes », s'avèrent satisfaire les besoins de reddition de sens des observations. Le troisième défi est de permettre à la recherche appliquée d'alimenter une recherche plus fondamentale. Nous l'avons souligné plus haut, la production de connaissances appliquées peut exiger de faire un détour par l'élaboration de cadres d'analyse inédits. Et finalement, comme nous l'avons suggéré plus haut, travailler sur la construction du médico-économique nécessite de développer des cadres analytiques, encore pauvres, pour « attraper » ce qui est supposé la favoriser, à savoir notamment l'utilisation des instruments et leurs conséquences, la transversalité et l'hybridation.

\section{Conclusion}

Dans cet article, nous avons cherché à cerner, à partir d'une analyse des travaux publiés de recherche en gestion, ce que l'on apprend de l'intégration du médical et de l'économique à l'hôpital public en France suite au déploiement, au cours des dix dernières années, de réformes ayant porté sur leur financement, leur gouvernance et leur organisation. Nous avons pour cela constitué un corpus de 67 publications, structuré autour des trois axes de déploiement des réformes : le design organisationnel et la délégation des responsabilités, l'instrumentation de gestion, et les acteurs dans les établissements.

Concernant le premier axe, de nombreux travaux ont porté sur l'organisation interne de l'hôpital et particulier sur la constitution et le fonctionnement des nouveaux centres de responsabilités dirigés par des médecins, les pôles. Ces travaux confirment que l'enjeu est 
de faire exister ces nouveaux centres de responsabilité en tant que lieu d'intégration du médical et de l'économique.

L'étude du second axe fait ressortir que les instruments de gestion déployés à l'hôpital pour accompagner les réformes ou dans le cadre de leur mise en œuvre n'ont pas permis en pratique de contribuer à cette intégration. L'enjeu médico-économique est en tant que tel rarement central dans les travaux étudiés, qui portent davantage les modalités de construction, d'utilisation et d'appropriation des nouveaux outils à l'hôpital.

Les travaux sur les acteurs au sein des établissements décrivent quant à eux des professionnels aux prises avec la gestion. Ils décrivent la manière dont les acteurs se saisissent des réformes ou l'impact de ces réformes sur les acteurs avec des résultats souvent très contrastés d'une publication à l'autre. À nouveau, la question de l'intégration du médical et de l'économique est rarement centrale dans ces travaux.

Il ressort de ces analyses que le médico-économique est, dans les travaux étudiés, plus un contexte qu'un enjeu d'étude en soi. En raison peut-être du caractère encore récent des réformes, ou des limites des approches méthodologiques et conceptuelles mobilisées pour aborder la question de la relation entre le médical et l'économique au sein des établissements, les résultats sur le processus de gestion de cette relation et ses effets restent eux-mêmes à construire. Les travaux étudiés soulignent cependant le poids de l'instrumentation de gestion au sein des établissements, et la grande variété des contextes et des pratiques.

Notre analyse conduit également à interroger nos propres pratiques de recherche et met en évidence un besoin de structuration accru des projets au sein de notre communauté, y compris pour identifier les thèmes et valoriser les résultats de travaux de recherche appliquée sur les établissements et leurs transformations.

Nous souhaitons donc que ce travail soit l'occasion d'engager une discussion avec le monde de la recherche et ses parties prenantes sur les voies de recherche futures comme sur la manière de les aborder. En effet, nous restons convaincus que la recherche en gestion peut, à côté des rapports produits par les différentes instances d'évaluation et de réflexion sur la transformation des hôpitaux et leur rôle au sein du système de soins, proposer des cadres méthodologiques et conceptuels susceptibles d'enrichir et de renouveler le questionnement sur des acteurs publics comme des praticiens sur l'hôpital et l'intégration en son sein du médical et de l'économique. Toutefois, les travaux sur la compréhension de ce processus et de ses effets ne doivent pas être exclusifs de mises en perspective plus distanciées, susceptibles de questionner l'émergence du médico-économique lui-même en tant qu'enjeu politique, social et gestionnaire, et d'aborder les questions de régulation d'un tel processus. Par conséquent, analyser la manière dont la relation entre le médical et l'économique a été abordée dans d'autres champs disciplinaires comme à l'articulation entre plusieurs champs compléterait utilement le travail que nous avons entrepris ici. 


\section{Bibliographie}

Aggeri F. et Labatut J., 2010. La gestion au prisme de ses instruments. Une approche généalogique des théories fondées sur les instruments de gestion. Finance Contrôle Stratégie 13 (3), 5-37.

Arndt M. and Bigelow B., 2006. Toward the Creation of an Institutional Logic for the Management of Hospitals: Efficiency in the Early Nineteen Hundreds. Med Care Res Rev. 63 (3), 369-94.

Baldvinsdottir G., Mitchell F. and Nørreklit H., 2010. Issues in the Relationship between Theory and Practice in Management Accounting. Management Accounting Research 21 (2), 79-82.

Battilana J. and Dorado S., 2010. Building Sustainable Hybrid Organizations: The Case of Commercial Microfinance Organizations. Academy of Management Journal 53 (6), 1419-1440.

Bérard E., 2013b. Rôle des instruments de gestion au sein des organisations : une approche sociomatérielle. Le cas de l'usage des comptes de résultat analytique et des budgets dans un hôpital public français. Thèse de doctorat. Université Panthéon-Sorbonne - Paris I; ESCP Europe.

Bérard E., Flachère I., Saulpic O. et Zarlowski P., 2015. Les outils financiers dans les hôpitaux : penser leur déploiement au-delà de la dimension technique. VI colloque santé et action sociale, Marseille.

Brunsson N., 1992. The Organization of Hypocrisy: Talk, Decisions and Actions in Organizations. Wiley, Chichester.

Busse R., Alexander G., Wilm Q. and Wiley M., 2011. Diagnosis-Related Groups in Europe: Moving Towards Transparency, Efficiency And Quality In Hospitals. McGraw-Hill Education, New York.

Denis J-L., Langley A. et Rouleau L., 2007. Strategizing in Pluralistic Contexts: Rethinking Theoretical Frames. Human Relations 60 (1), 179-215.

Domy P., Moulin G., Frechou D., Matrineau F et Müller C., 2014. Bilan et évaluation du fonctionnement des pôles dans les établissements de santé. Rapport des Conférences hospitalières, Mars

Doolin B., 1999. Casemix Management in a New Zealand Hospital: Rationalisation and Resistance. Financial Accountability\& Management 15 (3-4), 397-417.

Dumez H., 2010. Problèmes épistémologiques de la recherche qualitative. Le libellio d'Aegis 6 (4).

Giraud F., Saulpic O., Bonnier C., Delmond M-H., De Geuser F., Laulusa L. and Zrihen R., 2011. The Art of Management Control. Pearson Education France, Paris.

Glouberman S. and Mintzberg H., 2002. Gérer les soins de santé et le traitement de la maladie. Gestion 273 (3), 12-22.

Grenier C. et Bernardini J., (2015). Le manager hybride comme acteur-traducteur et acteur-clôture - Analyse du médecin-chef de pôle hospitalier. Revue Française de Gestion 250, 125-138.

Grenier C. et Bernardini J., 2016 (à paraître). External Change Implementation within Professional Organizations: Strategic and Enforced Logics Hybridization; An Agency View within French Hospitals and Universities In Pinheiro R., Ramirez F., Vrangbæk K. and Byrkjeflot H. (eds), Towards a Comparative Institutionalism? Forms, Dynamics, and Logics across the Organizational Fields of Health and Higher Education - Chapter 7, Emerald Group Publishing, London.

Hatchuel A., 2002. La théorie CK: Fondements et usages d'une théorie unifiée de la conception. Colloque Sciences de la conception, Lyon.

Jacobs K., 1995. Budgets: a Medium of Organizational Transformation. Management Accounting Research 6 (1), 59-75.

Jones C. S. and Dewing I. P., 1997. The Attitudes of NHS Clinicians and Medical Managers Towards Changes in Accounting Controls. Financial Accountability \& Management 13 (3), 261-280.

Kahn R. L., Wolfe D. M., Quinn R. P., Diedrick J. and Rosenthal R. A., 1964. Organizational Stress: Studies in Role Conflict and Ambiguity (Vol. XII). Wiley, Chichester.

Kitchener M., 2002. Mobilizing the Logic of Managerialism in Professional Fields: the Case of Academic Health Centre Mergers. Organization Studies 23 (3), 391-420. 
Kletz F., 2014. La tectonique des pôles. Annales des Mines - Gérer et comprendre 116 (2), 14-16.

Kraatz, M. and Block E., 2008. Organizational implications of institutional pluralism. The Sage Handbook of Organizational Institutionalism, Sage, 243-275.

Kurunmäki L., 2004. A Hybrid Profession: the Acquisition of Management Accounting Expertise by Medical Professionals. Accounting, Organizations and Society 29 (3-4), 327-347.

Lazarus R.S. and Folkman S., 1984.Stress Appraisal and Coping. Springer Publishing, New York.

Lenay O. and Moisdon J.-C., 2003. Du système d'information médicalisée à la tarification à l'activité. Trajectoire d'un instrument de gestion du système hospitalier. Revue Française de Gestion 146, 131-141.

Lewis M. W., 2000. Exploring Paradox: Toward a More Comprehensive Guide. Academy of Management Review 25 (4), 760-776.

Lowe A. and Doolin B., 1999. Casemix Accounting Systems: new Spaces for Action. Management Accounting Research 10 (3), 181-201.

Mazouz B., Garzon C. et Picard P., 2012. Les déviances dans les organisations publiques en quête de performance. Vers une gestion prophylactique des risques de déviance. Management International 16 (3), 92-100

Meah, 2009. Nouvelle gouvernance et comptabilité analytique par pôles. ANAP, Paris.

Moisdon J-C., 2008. Règles de gestion, outils, organisation. Le libellio d'Aegis 4 (1), 18-32.

Nicholson N., 1984. A Theory of Work Role Transitions. Administrative Science Quarterly 29 (2), $172-191$.

Or Z. et Renaud T., 2009. Principes et enjeux de la tarification à l'activité à l'hôpital (T2A). Enseignements de la théorie économique et des expériences étrangères. IRDES, Paris.

Pépin M. et Moisdon J-C., 2010. Les impacts de la T2A sur les modes d'organisation et de fonctionnement des établissements de santé, Dossiers Solidarité Santé (16).

Pouvourville (de) G., 2009a. L'organisation des soins In P.-L. Bras, G. de Pouvourville et D. Tabuteau, Traité d'économie et de gestion de la santé. Presses de Sciences Po, Paris, 401-406

Pouvourville (de) G., 2009b. Les hôpitaux français face au paiement prospectif au cas : la mise en œuvre de la tarification à l'activité. Revue Économique 60 (2), 457.

Reay T. and Hinings C.R., 2009. Managing the Rivalry of Competing Institutional Logics. Organization Studies $30,629-652$

Singly (de) C., 2009. Rapport de la Mission Cadres Hospitaliers. Ministère de la Santé et des Sports, Paris.

\section{Annexe \\ Corpus sur le Design organisationnel - références étudiées}

Angelé-Halgand N., 2012. Nouvelle gouvernance hospitalière : entre accountability et responsabilité. $I^{\text {er }}$ colloque ARAMOS, Paris.

Angelé-Halgand N., 2014. Manager pour réformer l'hôpital : les enjeux de l'efficience sans délégation In M. Djouldem, L. Imbeau, et C. De Visscher, Finances et politiques publiques : enjeux politiques et gestionnaires des réformes. Bruylant, Paris, 159-186

Bérard E., 2013a. Les pôles d'activité à l'hôpital vus comme un système cybernétique : une analyse en termes de contrôle de gestion. Journal de gestion et d'économie médicales 31 (4), 187-205.

Bréchat P-H., Leenhardt A., Mathieu-Grenouilleau M-C., Rymer R., Matisse F., Baraille D. et Beaufils P., 2010. Des pôles d'activités hospitaliers entre gestion de la crise et mise en œuvre d'une politique de santé publique. Revue de Santé Publique 5, 571-580.

Crozet P., Kaaniche A. et Lienard J., 2008. Nouvelle gouvernance à l'hôpital : recomposition de l'organisation et gestion des ressources humaines. Politiques et management public 26 (2), 31-52.

Dos Santos C., Mousli M. et Randriamiarana J. , 2014, . De la T2A à HPST : quelle contribution du contrôle de gestion au pilotage de la performance ? Étude de cas d'un CHU. Journal de gestion et d'économie médicales 32 (4), 291-307. 
Flachère I., 2014. Management accounting tools as mediators of social reconfiguration in healthcare organizations. II congrès ARAMOS, Marseille.

Gavault S., Laude L. et Baret C., 2014. L’institutionnalisation des pôles d'activité médicale entre agir stratégique et agir projectif. IIème Congrès ARAMOS, Marseille.

Gouffé V. et Cargnello-Charles E., 2014. Élaborer et piloter des projets dans les organisations sanitaires et médico-sociales : acteurs, savoirs, outils. II Congrès ARAMOS, Marseille.

Grenier C. et Bernardini J., 2013. La décision du chef de pôle hospitalier aux prises avec des logiques institutionnelles concurrentes : vers un espace hybride décisionnel. Conférence GEAP, Edimbourg.

Grenier C., Bernardini J. and Zeller C., 2014. External Change Appropriation within Professional Organization: Strategic and Uncontrolled Logics Hybridization-an Agency View within French Hospital and University. 30th EGOS Colloquium, Rotterdam.

Guicheteau J. et Maestre-Levèvre A., 2009. Un tableau de bord équilibré au service du pilotage médicoéconomique. Revue Hospitalière de France 351, 48-50.

Havard C., 2013. La mise en place d'une organisation polaire au sein d'un centre hospitalier : une approche par l'effectivité. $X X^{\mathrm{e}}$ congrès AGRH, Paris.

Havard C., 2014. La mise en place des pôles au sein d'un centre hospitalier : quelle articulation des logiques ? II congrès ARAMOS, Marseille.

Lartigau J., 2009. L'évolution de la fonction contrôle de gestion à l'hôpital. Journal de gestion et d'économie médicales 27 (7), 371-391.

Moisdon J-C., 2008. Règles de gestion, outils, organisation. Le libellio d'Aegis 4 (1), 18-32.

Nobre T. et Lambert P., 2012. Le management de pôles à l'hôpital-Regards croisés, enjeux et défis. Dunod, Paris.

Vallejo J., Sampieri-Teissier N. et Baret C., 2014. Analyse du processus de délégation des pôles d'activité médicale : une approche par la sociologie de la traduction. II Congrès ARAMOS, Marseille.

Vincent B. et Montalan M-A., 2014. Facteurs clés de la performance collective de groupements d'achats de produits de santé. Journal de gestion et d'économie médicales 32 (1), 11-24.

\section{Corpus sur les instruments - références étudiées}

Angelé-Halgand N. et Garrot T., 2014. Hôpital, réformes et outils de gestion: l'émergence d'un gouvernement à distance. II colloque ARAMOS, Marseille.

Arthus I., Montalan M-A. et Vincent B., 2009. Quels outils pour piloter la performance d'une Équipe Mobile de Gériatrie ? Journal d'économie médicale 27 (1), 43-57.

Bérard E., 2011. L'appropriation du dispositif de contractualisation interne à l'hôpital de V : évolution conjointe de la stratégie et du contrôle. 32 ${ }^{\mathrm{e}}$ Congrès de l'Association Francophone de Comptabilité, Montpellier

Bérard E., Saulpic O. and Zarlowski P., 2013. Accounting for the Influence of Management Devices in the Evolution of Public Organizations Practices: a Sociomaterial Perspective. 30th EGOS Colloquium, Rotterdam.

Bérard E. et Steyer V., 2013. La responsabilité contractuelle à l'hôpitalLa responsabilité contractuelle à l'hôpital : un modèle fonctionnel malgré tout? Revue française de gestion 237, 147-167.

Bonnier C., Flachère I., Saulpic O. et Zarlowski P., 2013. Le déploiement d'outils financiers dans le cadre de la réforme du système de soins : quand les acteurs utilisent les outils parce que c'est ce qu'il convient de faire. Colloque de la revue Politiques et Management Public, Paris.

Bonnier C., Saulpic O. et Zarlowski P., 2013. Un tableau de bord objet-frontière entre deux communautés : étude de cas d'une clinique. $34^{\mathrm{e}}$ Congrès de l'Association Francophone de Comptabilité, Montréal.

Capelletti L., Lartigau J. et Noguéra F., 2013. La modernisation du pilotage des RH dans la fonction publique hospitalière : cas de la gestion de la masse salariale. $24^{\mathrm{e}}$ Congrès de l'AGRH, Paris.

Cauhape J. et Raveneau V., 2008. Mise en place d'un système décisionnel informatisé : vers un outil de pilotage commun aux pôles d'activité et à la direction. Journal d'Économie Médicale 26 (1), 79-86.

Colasse S. et Nakhla M., 2011. Les démarches de contractualisation comme processus de conception L'émergence du contrôle de gestion médicalisé à l'hôpital. Politiques et Management Public 28 (3) 311-331. 
Crémieux F., Saulpic O. et Zarlowski P., 2013. L'introduction de comptes de résultats de pôles dans un hôpital public : analyses et propositions à partir d'une étude de cas. Journal de gestion et d'économie médicales 30 (7), 419-434.

Dumas M., Douguet F. et Muñoz J., 2012. L'appropriation d'un outil de la qualité des soins à l'hôpital. Journal de gestion et d'économie médicales 30 (3), 127-149.

Flachère I., 2014. Management Accounting Tools as Mediators of Social Reconfiguration in Healthcare Organizations. II congrès ARAMOS, Marseille.

Georgescu I., 2012. Communication de l'information et pression financière interne: une étude exploratoire dans une organisation professionnelle. 33 Congrès de l'Association Française de comptabilité, Grenoble.

Georgescu I. et Hartmann F.G., 2011. Explaining Data-Manipulation by Physicians: 'Tell me who Presses you and I will Predict your Manipulation'. 34 ${ }^{\text {th }}$ Annual congress European Accounting Association, Rome.

Georgescu I. et Naro G., 2012. Pressions budgétaires à l'hôpital: une étude qualitative du concept de «RAPM » auprès de praticiens hospitaliers. Comptabilité-Contrôle-Audit 18 (3), 67-95.

Gouffé V. et Cargnello-Charles E., 2013. Les organisations de santé et leurs environnements institutionnels : réceptacles d'injonctions ou acteurs stratégiques. $V^{\mathrm{e}}$ colloque Santé et Action sociale, Marseille.

Grévin A., 2010. Le jeu avec les outils de gestion comme nouvelle compétence stratégique. Un établissement de santé face aux régulations du secteur sanitaire. Journal d'Économie Médicale 28 (8), 329-345.

Jeanblanc G. et Durand-Zaleski I., 2008. Évaluation des pratiques professionnelles et indicateurs de performance. Journal d'Économie Médicale 26 (4), 218-228.

Juven P-A., 2013. Codage de la performance ou performance du codage. Journal de Gestion et d'Economie Médicale $31(2 / 3)$.

Kauffmann V., 2008. Pôles d'activité médicale et contractualisation aux Hospices Civils de Lyon : Bilan et perspectives. Journal de gestion et d'économie médicales 26 (6), 351-359.

Le Pogam L., Luangsay-Catelin C. et Notebaert J.-F., 2009. La performance hospitalière : à la recherche d'un modèle multidimensionnel cohérent. Management \& Avenir 25 (5), 116-134.

Margarit C. et Peyret P., 2008. Nouvelle gouvernance et comptabilité analytique par pôles. Journal de gestion et d'économie médicales 26 (1), 48-55.

Marty M-C., Wable E., Debonne J-M., Dumond J-P., Koenig G. et Vandangeon I., 2012. L'évaluation de l'appropriation du projet d'établissement de 1'HIA Val de Grace. ${ }^{\text {er }}$ congrès Aramos, Paris.

Mel T., 2013. Évaluation de la performance des centres hospitaliers: quels outils au regard du nouveau management Public? 5 Colloque santé, Marseille.

Moisdon J.-C., 2012. Le paradoxe de la boîte noire. Réformes hospitalières et organisation. Droit et Société, (1), 91-115.

Montalan M-A. et Vincent B., 2011. Élaboration d'un balanced scorecard en milieu hospitalier. Revue française de gestion 211, 93-102.

Nobre T. et Haouet I., 2011. Le cas d'un balanced scorecard en contexte hospitalier. Revue française de gestion 211, 103-118

Nobre T. et Signolet, 2009. Le système d'information de gestion hospitalier : quelles caractéristiques privilégier pour favoriser le développement des outils de gestion? $30^{\mathrm{e}}$ Congrès de l'Association Française de comptabilité, Strasbourg.

Pascal C., Capgras J-B., Guilhot N. et Claveranne J.-P., 2011. L’information médico-économique entre décision stratégique et action publique : tout savoir pour peu pouvoir! Politiques et management public 28 (1), 57-82.

\section{Corpus sur les acteurs - références étudiées}

Beaucourt C. et Laude L., 2010. Diriger, une analyse de la relation dynamique entre compromis et transgression. $X X^{\mathrm{e}}$ Congrès de l'AGRH, Rennes/Saint-Malo.

Burellier F. et Valette A., 2011. Garder ou tomber la blouse? Analyse de la transition vers le rôle de médecingestionnaire. Journal de gestion et d'économie médicale 29 (6-7), 336-345. 
Bouret, P., 2008. Encadrer dans la fonction publique hospitalière : un travail de lien invisible. Revue française d'administration publique 128 (4), 729-740.

Coulon R., 2011. Le cadre de santé, la gestion et le soin. XXII Congrès de l'AGRH.

De Pouvourville G., 2010. La crise d'identité des médecins face au nouveau management de l'hôpital. Le journal de l'école de Paris du management 86 (6), 22-29.

Detchessahar M. et Grevin A., 2009. Un organisme de santé... malade de «gestionnite ». Annales des Mines Gérer et comprendre 98 (4), 27-37.

Divay S. et Gadea C., 2008. Les cadres de santé face à la logique managériale. Revue française d'administration publique 128 (4), 677.

Duchamp D. et Persson S., 2012. Vers des Médecins-Managers-Mentors? Premiers résultats d'un accompagnement concerté dans un CHU. Management \& Avenir 53 (3), 142-161.

Dumas M. et Ruiller C., 2013. «Être cadre de santé de proximité à l'hôpital », quels rôles à tenir? Revue de gestion des ressources humaines 87 (1), 42-58.

Fiore C. et Sampieri-Teissier N., 2011. Transformer les chefs de pôles hospitaliers en véritables pilotes de la performance. Quelles modalités pour un changement de posture? $V^{\mathrm{e}}$ colloque santé et action sociale, Marseille.

Fray A.M., 2009. Nouvelles pratiques de gouvernance dans le secteur hospitalier : conséquences managériales sur les acteurs. Management et avenir 28, 152-159.

Georgescu I. et Commeiras N., 2012. Pression budgétaire et implication organisationnelle des médecins. Journal de gestion et d'économie médicales 29 (6), 294-304.

Georgescu I., et Rivière A., 2013. Evolution des pratiques chez les acteurs du soin à l'hôpital, managérialisation et identité professionnelle. XXIV congrès AGRH, Paris.

Grenier C., Valette A., Burrelier F. and Bernardini J., 2013. Hybrids in Public Organizations: a Compartmentalized Appropriation of a (Expected) Hybrid Role. $29^{\text {th }}$ EGOS colloquium, Montréal.

Krief N., 2012. Les préoccupations des acteurs hospitaliers en question, analyse croisée et dynamique de construction collective. @grh 2 (3), 43-67.

Rivière A., Commeiras N. et Loubes A., 2013. Tensions de rôle et stratégies d'ajustement : une étude auprès de cadres de santé à l'hôpital. Journal de gestion et d'économie médicales 31 (2), 142-162.

Valette A., Fatien P. et Burellier F., 2011. Entre le stéthoscope et le compte de résultat : que disent les médecinsgestionnaires sur leur gestion des paradoxes ? AIMS, Nantes.

Valette A. et Burellier F., 2014. Quand l'habit fait le moine les chefs des pôles hospitaliers : devenir des hydrides «malgré tout »? Annales des Mines - Gérer et comprendre 116 (2), 4-13. 